\begin{tabular}{lllll} 
Abstract 222 Table 1 & & & & \\
\hline & Correlation coefficient & $p$ & RR & $95 \%$ Cl \\
\hline ESR & 0,34 & $<0,05$ & 1,56 & $0,36-0,87$ \\
ANA & 0,22 & $>0,05$ & 1,12 & $0,43-1,50$ \\
AntiDNA Ab & 0,13 & $>0,05$ & 0,98 & $0,67-2,13$ \\
Low Hb level & 0,48 & $<0,05$ & 1,99 & $0,45-0,80$ \\
Low leucocytes & 0,23 & $>0,05$ & 1,01 & $0,56-1,33$ \\
Low lymphocytes & 0,56 & $<0,05$ & 2,05 & $0,33-0,67$ \\
Antiphospholipid syndrome & - & - & 2,30 & $0,61-0,88$ \\
Pulmonary Involvement (SLAM) & - & - & 1,88 & $0,23-0,82$ \\
\hline
\end{tabular}

223 SERUM 25-HYDROXYVITAMIN D3 LEVELS AND CLINICAL COURSE OF SYSTEMIC LUPUS ERYTHEMATOSUS : A CROSS SECTIONAL RETROSPECTIVE COHORT STUDY IN A REFERRAL CENTRE

${ }^{1,2}{ }^{2} \mathrm{M}$ Mohd Noh*, ${ }^{2} \mathrm{DM}$ Suahilai, ${ }^{2} \mathrm{NS}$ Shahril, ${ }^{3} \mathrm{SA}$ Alias. ${ }^{1}$ University Malaysia Sabah, Faculty Of Medicine andAllied Health Sciences, Kajang, Malaysia; ${ }^{2}$ Hospital Putrajaya, Medicine, Putrayaja, Malaysia; ${ }^{3}$ Hospital Putrajaya, Medical, Putrajaya, Malaysia

\subsection{6/lupus-2017-000215.223}

Background and aims To study the association between serum 25-Hydroxyvitamin D3 levels and clinical manifestation, disease activity, and disease damage of systemic lupus erythematosus (SLE).

Methods This was a retrospective cross sectional study of SLE patients seen between 1996 until 2015. Patients were grouped according to the Vitamin D3 levels : group $1(<25 \mathrm{nmol} / \mathrm{L}$ : deficiency), group $2(25-75 \mathrm{nmol} / \mathrm{L}$ : insufficiency) and group 3 (>75 nmol/L : adequate). Assessment of disease activity was done using Systemic Lupus Erythematosus Disease Activity Index Selena Modification (SLEDAI) while Systemic Lupus International Collaborating Clinics (SLICC) was use for disease damage.

Results A total of 42 patients had their serum 25-Hydroxyvitamin D3 levels taken at one point of their visit. Majority were females $(n=41)$. Mean age was 37.2 years $(S D \pm 13.13)$ and mean duration of illness 9.5 years $(\mathrm{SD} \pm 5.7)$. The proportion of patients with 25-Hydroxyvitamin D3 level group 1 was $31 \%$, group 2 was $61.9 \%$ and group 3 was $7.1 \%$ respectively. Main clinical manifestations were haematological $71.1 \%$, arthritis $68.9 \%$, malar rash $53.3 \%$. SLEDAI mild activity (0-3) $90.5 \%$, moderate activity (4-8) was $4.8 \%$ and severe activity $(>8)$ was $4.8 \%$. SLICC showed $78.6 \%$ had no damage and $21.4 \%$ with damage. Test of association using ANOVA, did not show any significance between Vitamin D3 level and SLEDAI, SLICC and clinical manifestations were observed among the group.

Conclusions Vitamin D insufficiency and deficiency was common in our SLE cohort.However, we did not find significant association between vitamin D deficiency and disease activity, damage or clinical manifestations. The study limitation includes small number of patients and retrospective design.

\section{PROTEIN-LOSING ENTEROPATHY AND FULMINANT INTESTINAL VASCULITIS IN A FILIPINO LUPUS PATIENT: A CASE REPORT}

RM Molina, S Navarra. University of Santo Tomas, Internal Medicine- Section of Rheumatology, Metro Manila, Philippines

\subsection{6/lupus-2017-000215.224}

Background and aims We describe a rare case of GI vasculitis flare in SLE presenting as diarrhoea, hematochezia and profound hypoalbuminemia.

Methods Case report

Results A 49 year old patient has had stable SLE in the past 22 years until she developed episodes of diffuse abdominal pains accompanied by alternating diarrhoea with constipation for 12 months; colonoscopy showed rectal ulcers and abdominal CT scan showed colonic diverticulosis. She received supportive therapy with only minimal relief. She was first admitted due to severe abdominal pain and worsening diarrhoea; laboratory tests disclosed thrombocytopenia, hypocomplementemia and high titer anti-dsDNA; there was dramatic resolution of symptoms with high dose corticosteroid and she was discharged significantly improved. A few weeks later while on tapering prednisone, she was re-admitted because of recurrence of profuse diarrhoea with severe electrolyte imbalance. Hospital course was marked by diarrhoea, severe hypoalbuminemia with progressive anasarca requiring intravenous albumin infusions, and episodes of massive hematochezia requiring multiple blood transfusions. Colonoscopy showed ischaemic colitis with edematous friable recto-sigmoid mucosa. Intravenous corticosteroid was increased. She underwent abdominoperineal resection with ileal resection of necrotic intestinal segments; histopath confirmed haemorrhagic gangrenous necrosis of the small intestine and colon, with small and medium vessel vasculitis and thrombosis. Although immediate post-operative course was uneventful, she succumbed a few days later to fulminant bacterial peritonitis due to anastomotic failure with extension of the bowel ischemia.

Conclusions This case illustrates the diagnostic dilemma and management challenges of lupus mesenteric vasculitis, requiring intensive monitoring for complications with aggressive supportive and disease-specific measures.

\section{GASTROINTESTINAL FLARES AMONG FILIPINO PATIENTS WITH SLE: A CASE SERIES}

RM Molina*, S Navarra. University of Santo Tomas, Internal Medicine- Section of Rheumatology, Metro Manila, Philippines

\subsection{6/lupus-2017-000215.225}

Background and aims Gastrointestinal (GI) involvement in SLE ranges from $2.2 \%-9.7 \%$, and nonspecific manifestations pose a diagnostic challenge. This series describes characteristics, treatment and outcomes of patients with GI involvement as the primary manifestation of SLE activity.

Methods Case series of 10 Filipino SLE patients with proven GI flares, seen at the Lupus Clinics of University of Santo Tomas (UST) Hospital, Manila, Philippines.

Results All 10 patients were females with mean age 31.7+9.35 SD $(19-49)$ and disease duration 6.08 \pm 7.34 SD (0 - 22) years. Most commonGI manifestations were abdominal pain (100\%), ileus (60\%), vomiting (50\%) and diarrhoea(40\%). Extra-intestinal manifestations included malar rash (70\%), arthritis (60\%), 
Abstract 225 Table 1 Disease characteristics, treatment and outcome of 10 SLE patients with GI manifestations

\begin{tabular}{|c|c|c|c|}
\hline Age & Gastrointestinal manifestations or involvement & Treatment & Outcome \\
\hline 34 & Abdominal pain, nausea, vomiting, diarrhea & Dexamethasone & Improved \\
\hline 33 & $\begin{array}{l}\text { Recurrent abdominal pain, abdominal tenderness, atrophic } \\
\text { gastritis, appendicitis }\end{array}$ & $\begin{array}{l}\text { Methylprednisolone, } \\
\text { cyclophosphamide, explor } \\
\text { lap with appendectomy }\end{array}$ & Improved \\
\hline 45 & $\begin{array}{l}\text { Epigastric pain, abdominal tenderness, ileus with bowel } \\
\text { dilatation }\end{array}$ & Dexamethasone & Improved \\
\hline 49 & $\begin{array}{l}\text { Abdominal pain, diarrhea, hematochezia, ileus, "double } \\
\text { halo" sign by CT scan, rectal ulcers, necrotic rectosigmoid }\end{array}$ & $\begin{array}{l}\text { Hydrocortisone, } \\
\text { dexamethasone, } \\
\text { abdominoperineal with ileal } \\
\text { resection }\end{array}$ & Died \\
\hline 25 & Abdominal pain, vomiting, ileus & Hydrocortisone & Improved \\
\hline 33 & Abdominal pain, nausea, vomiting, ileus & Methylprednisolone & Improved \\
\hline 27 & Abdominal pain, diarrhea & $\begin{array}{l}\text { Hydrocortisone, } \\
\text { dexamethasone }\end{array}$ & Improved \\
\hline 19 & $\begin{array}{l}\text { Abdominal pain, vomiting, ileus, "double halo" and "comb" } \\
\text { sign by CT scan }\end{array}$ & Methylprednisolone & Improved \\
\hline 24 & $\begin{array}{l}\text { Abdominal pain, vomiting, diarrhea, mucosal inflammation, } \\
\text { pneumoperitoneum, by CT scan, ileal perforation }\end{array}$ & $\begin{array}{l}\text { Methylprednisolone, } \\
\text { belimumab, ileal resection }\end{array}$ & Improved \\
\hline 28 & $\begin{array}{l}\text { Abdominal pain, ileus, ascites, diffuse enterocolitis with } \\
\text { pancreatitis by CT scan }\end{array}$ & $\begin{array}{l}\text { Methylprednisolone, } \\
\text { cyclophosphamide }\end{array}$ & Improved \\
\hline
\end{tabular}

hypocomplementemia (60\%), alopecia (50\%), and hemolyticanemia (40\%). All patients showed significant initial response to high dose corticosteroid. Three patients eventually required surgery including ileal resection, abdomino-perineal resection and appendectomy; post-op histopath findings confirmed vasculitis in all 3 patients. One patient with ileal ischemia and perforation requiring resection also received belimumab infusions which enabled successful tapering and discontinuation of steroid. Another patient with refractory protein losing enteropathy and ischaemic colitis underwent abdomino-perineal with ileal resection, but succumbed to anastomotic failure with fulminant bacterial peritonitis.

Conclusions Though rare, gastrointestinal flare in SLE can be potentially catastrophic. Because of nonspecific manifestations, diagnosis strongly relies on clinical assumption and response to steroids. In some cases, surgery can be life-saving and belimumab offers another effective therapeutic option.

\section{RELAPSES OF LUPUS NEPHRITIS - RISK FACTORS, INCIDENCE AND IMPACT OF OUTCOME}

${ }^{1} \mathrm{D}$ Monova, ${ }^{2} \mathrm{~S}$ Monov*. 'Medical University - Sofia- Medical Institute, Department of Internal Diseases, Sofia, Bulgaria; ${ }^{2}$ Medical University - Sofia, Department of Internal Diseases- Clinic of Rheumatology, Sofia, Bulgaria

10.1136/lupus-2017-000215.226
Background and aims The aim of this study was to review renal flare frequency, to identify potential risk factors for relapses, to assess the value of serological tests during flares and to analyse their impact of global outcome in lupus nephritis (LN) patients.

Methods Patients with biopsy proven LN were identified from our database. LN classes were defined according to the ISN/ RPS classification. According to the response to treatment, LN patients were divided into 3 groups of complete remission (CR), partial remission (PR) and no response (NR). Those in remission were divided into 2 groups of relapsing and nonrelapsing during maintenance period.

Results $218(70,64 \%)$ of 276 SLE patients with biopsy proven LN (class I-18 patients, class II-45, class III-56, class IV-75, class V-54, class VI-2, mixed forms - 26) achieved either CR $(55,8 \%)$ or PR $(23,2 \%) .47$ patients had one flare, 36 - two, 27 - three, $17 \geq 4$ flares. The maintenance immunomodulating drugs at the time of flare was low dose corticosteroids and/or azathioprine. Non-adherence to treatment at time of relapse was documented in 26 patients.

Conclusions Renal flares in patients with LN are common, have a negative impact on outcome, but cannot be readily predicted. Our study shows that $58,83 \%$ of $\mathrm{LN}$ patients develop at least one relapse after reaching remission, usually within 2 years. The length of time to flare tends to be shorter in cases of preceding PR than in CR. Lack of adherence to long term immunosuppression was identified as a significant factor in LN flare $(20,47 \%)$. 